\title{
Convection-induced nonlinear symmetry breaking in wave mixing
}

\author{
Roberta Zambrini, ${ }^{1}$ Maxi San Miguel, ${ }^{2}$ Céline Durniak, ${ }^{3}$ and Majid Taki ${ }^{3}$ \\ ${ }^{1}$ Department of Physics, University of Strathclyde, Glasgow, G4 ONG, United Kingdom \\ ${ }^{2}$ Instituto Mediterráneo de Estudios Avanzados, IMEDEA (CSIC-UIB), Campus Universitat Illes Balears, \\ E-07071 Palma de Mallorca, Spain \\ ${ }^{3}$ Laboratoire de Physique des Lasers, Atomes et Molécules, CNRS UMR 8523, Centre d'Etudes et de Recherches Lasers et Applications, \\ Université des Sciences et Technologies de Lille, 59655 Villeneuve d'Ascq Cedex, France
}

(Received 18 January 2005; published 19 August 2005)

\begin{abstract}
We show that the combined action of diffraction and convection (walk-off) in wave mixing processes leads to a nonlinear symmetry breaking in the generated traveling waves. The dynamics near to threshold is reduced to a Ginzburg-Landau model, showing an original dependence of the nonlinear self-coupling term on the convection. Analytical expressions of the intensity and velocity of traveling waves emphasize the utmost importance of convection in this phenomenon. These predictions are in excellent agreement with the numerical solutions of the full dynamical model.
\end{abstract}

DOI: 10.1103/PhysRevE.72.025603

PACS number(s): 42.65.Tg, 89.75.Kd, 42.65.Sf, 47.54.+r

There is currently a considerable interest in understanding the role of convection (drift) in pattern forming systems [1-4] in such diverse fields as hydrodynamics [5], plasma physics [6], traffic flow [7], crystal growth [8], and nonlinear optics [9]. The most important and common result in these studies is that convection, modeled by gradient terms $(\vec{\alpha} \cdot \vec{\nabla}$ with $\vec{\alpha}$ group velocity), breaks the reflection symmetry $(\vec{r}$ $\rightarrow-\vec{r}$ ) and dramatically affects pattern selection in spatially extended systems. This is a linear symmetry breaking where convection terms are generally considered to have the only effect to induce a traveling character to selected patterns, and to induce a peculiar regime of convective instability [2]. It has been largely studied how the existence, the type, and the dynamics of the pattern selected are closely related to the linear transition from convective instability (propagation overcomes amplification of perturbations) to absolute instability (amplification dominates).

In contrast, in this paper, we discuss an unforeseen effect of convection in the dynamics of spatially extended systems that does not rely on such a transition. Here, we show how convection, that is a linear phenomenon, actually modifies the intrinsic nonlinearity of the system. More precisely, we show that convection affects the nonlinear mode interaction at onset of the instability leading to a nonlinear symmetry breaking in the generated, otherwise symmetrical, traveling waves. Our analytical description of this mechanism, based on the amplitude equation of the degenerate optical parametric oscillator (DOPO), demonstrates an original dependence of the nonlinear self-coupling term upon convection. Although we present our investigations in the context of optics, we believe that our result is generic for spatially extended systems with convection and characterizes the key role of convection in the nonlinear dynamics of such systems.

In a DOPO degenerate optical parametric oscillator a laser beam is injected in a cavity containing a nonlinear (quadratic) crystal and a signal beam with half the pump frequency is generated. When the signal has polarization orthogonal to the pump the DOPO is in type-I phase matching. It is well known that, due to the birefringence of the crystal, the Poynting vectors of pump and signal fields are not parallel and walk-off each other [10]. Therefore convection (walk-off) arises naturally in these devices. Degenerate optical parametric oscillators are also at the basis of interesting quantum phenomena, stemming from their quadratic nonlinearity, as, for instance, entanglement between off-axis modes [11]. It has been shown that the walk-off strongly influences such twin beam correlations: in the convective regime (induced by walk-off) the entanglement is destroyed by macroscopic amplification of quantum noise [12]. Increasing the pump intensity, an absolutely stable traveling pattern arises in the signal and the entanglement is restored. Still, important walk-off effects are observed, as one of the twin beams is more intense and fluctuates more than the opposite one [13].

We start from the description of a type-I DOPO in the mean-field approximation including diffraction and walk-off [10],

$$
\begin{gathered}
\partial_{t} A_{p}=\gamma_{p}\left[-\left(1+i \Delta_{p}\right) A_{p}+i a_{p} \nabla_{\perp}^{2} A_{p}-A_{s}^{2}+E_{0}\right] \\
\partial_{t} A_{s}=\gamma_{s}\left[-\left(1+i \Delta_{s}\right) A_{s}+i a_{s} \nabla_{\perp}^{2} A_{s}+A_{p} A_{s}^{*}-\alpha_{s} \partial_{x} A_{s}\right],
\end{gathered}
$$

where $A_{p}$ and $A_{s}$ are the normalized slowly varying envelopes for pump and signal fields, respectively. In these equations, the diffraction is described by $\nabla_{\perp}^{2}=\partial_{x}^{2}+\partial_{y}^{2}$ where $(x, y)$ are the spatial transverse coordinates. The parameters $\Delta_{p, s}$, $\gamma_{p, s}$, and $a_{p, s}$ are the detunings, the cavity decay rates, and the diffraction coefficients, respectively. $E_{0}$ is the normalized external pump and $\alpha_{s}$ is the signal walk-off coefficient that characterizes convection in this system. We stress that the walk-off cannot be eliminated from Eqs. (1) by a change of reference frame, being relative between pump and signal.

Both convective and absolute instabilities have been reported for the stationary solution $A_{p}=E_{0} /\left(1+i \Delta_{p}\right), A_{s}=0[10]$. We just recall that for the case $\Delta_{s}<0$ and $\Delta_{s} \Delta_{p}-1<0$, which we are interested in, degenerate OPOs exhibit a supercritical bifurcation at $E_{0}^{c}=\sqrt{1+\Delta_{p}^{2}}$. This is the linear threshold at which stationary homogeneous solutions become unstable 
to traveling wave perturbations with wave vectors $\vec{k}$ $=\left(k_{x}, k_{y}\right)$ with modulus $k=k_{c}=\sqrt{-\Delta_{s} / a_{s}}$ and frequency $\omega_{c}=$ $-\gamma_{s} \alpha_{s} k_{c}$. Under periodic boundary conditions the convective instability is suppressed and the traveling rolls arising at the signal generation threshold are absolutely stable.

To study the role of convection in the nonlinear symmetry breaking of the generated traveling waves, and to keep mathematics as simple as possible, we perform the reduction of the model (1) into a single Ginzburg-Landau (GL) equation valid close to threshold of the DOPO emission. In the sequel we set $\mu_{p}=E_{0} /\left(1+i \Delta_{p}\right)$ with $\mu=\left|\mu_{p}\right|=E_{0} / \sqrt{1+\Delta_{p}^{2}}$, and $B$ $=A_{p}-\mu_{c}$ where $\mu_{c}=1$. We expand the signal as $A_{s}=\varepsilon A^{(1)}$ $+\varepsilon^{2} A^{(2)}+\varepsilon^{3} A^{(3)}+\cdots$ with a similar expansion of the pump. The small parameter $\varepsilon$ measures the distance from the DOPO emission threshold: $\varepsilon^{2}=\mu-\mu_{c}$. This fixes the choice of multiple scales for $t$ and $x$ to $t=T_{0}+\epsilon T_{1}+\epsilon^{2} T_{2}+\cdots, x=X_{0}+\epsilon X$, and $y=Y_{0}+\sqrt{\epsilon} Y$ (see Refs. [14] for details). The temporal and spatial derivatives in Eqs. (1) are then replaced by $\partial_{t}=\partial_{T_{0}}$ $+\epsilon \partial_{T_{1}}+\epsilon^{2} \partial_{T_{2}}+\cdots, \quad \partial_{x}=\partial_{X_{0}}+\epsilon \partial_{X}, \quad \partial_{y}=\partial_{Y_{0}}+\sqrt{\epsilon} \partial_{Y}$. We set $A^{(1)}=A \exp i\left(\omega_{c} T_{0}+k_{c} X_{0}\right)+A^{*} \exp -i\left(\omega_{c} T_{0}+k_{c} X_{0}\right)$, that is a wave traveling in the walk-off direction, with $A$ and its complex conjugate $A^{*}$ slowly varying amplitudes. By substitution of the above expansion of the signal $A_{s}$ in Eqs. (1) and using the new scalings and derivatives, a hierarchy of equations for each successive correction is obtained. Applying the solvability condition at order $O\left(\varepsilon^{3}\right)$ we get the following amplitude equation, which describes the evolution of the signal written in the scaled time $\tau=\gamma_{s} t$, and in the variable $S=\varepsilon A$ :

$$
\partial_{\tau} S+\alpha_{s} \partial_{x} S=(\mu-1) S-2 a_{s} \Delta_{s} \mathcal{L}_{x y}^{2} S-\beta|S|^{2} S
$$

with

$$
\beta=\frac{2}{1+\Delta_{p}^{2}}+\frac{C^{2}+1+D^{2}+i D\left(C^{2}-1-D^{2}\right)}{\left[1+(C+D)^{2}\right]\left[1+(C-D)^{2}\right]} .
$$

We have set $\mathcal{L}_{x y}=\left(\partial_{x}+\partial_{y}^{2} / 2 i k_{c}\right)$ in Eq. (2) and $C=\Delta_{p}+4 a_{p} k_{c}^{2}$ and $D=2 \omega_{c} / \gamma_{p}=-2 \alpha_{s}\left(\gamma_{s} / \gamma_{p}\right) k_{c}$ in the expression of the nonlinear self-coupling coefficient $\beta$. In the absence of convection $\left(\alpha_{s}=0\right)$, the parameter $D$ vanishes and the expression of $\beta$ greatly simplifies to $\beta=2 /\left(1+\Delta_{p}^{2}\right)+1 /\left(1+C^{2}\right)$ and stationary rolls arise in the signal profile [15]. We show here that the presence of convection drastically affects the pattern formation mechanism with respect to both linear and, more importantly, nonlinear dynamics. Indeed, the most important result is that the parameter $D$, that characterizes convection, strongly modifies the nonlinear self-coupling term $\beta$. It affects the saturation term $[\operatorname{Re}(\beta)]$ and induces intrinsic nonlinear phase modulations $[\operatorname{Im}(\beta)]$. This result is in contrast with almost all previous studies of model equations describing the near-threshold dynamics such as the GinzburgLandau or Swift-Hohenberg equations where the convection only yields to the propagation term of Eq. (2).

At this stage one has to notice that the presence of convection via the nonzero $\operatorname{Im}(\beta)$ breaks the well-known variational form of the GL Eq. (2) in its one-dimensional version (where $\mathcal{L}_{x y}=\partial_{x}$ ), since all the remaining coefficients are real [16]. As a consequence Eq. (2), obviously, cannot exhibit stationary homogeneous solutions (or stationary rolls). More- over, the nonvariational effect leads to (i) a symmetry breaking in the opposite traveling waves and (ii) an excess velocity (with respect to convection velocity) in these waves stemming from the nonlinear frequency modulation. Both points are analytically characterized below.

Let us find the solutions of Eq. (2), corresponding to the nonlinear saturated selected modes, in the form $S_{s t}$ $=S_{0} \exp i(\Omega \tau+k x)$. They read $\left|S_{0}\right|^{2}=\left(\mu-1+2 a_{s} \Delta_{s} k^{2}\right) / \operatorname{Re}(\beta)$ and $\Omega=-\alpha_{s} k-\operatorname{Im}(\beta)\left|S_{0}\right|^{2}$, and represent the leading contribution to the fundamental modes $\left( \pm k_{c}\right)$. This leading contribution is not sufficient since the total intensity of each mode is now fixed during their nonlinear interaction induced by the convection. For our purpose, to characterize the nonlinear symmetry breaking, we need to take into account the contributions up to the third order in $\varepsilon$. This can be achieved by solving the hierarchy of the inhomogeneous linear problems, at each order in $\varepsilon$, by means of Fredholm alternative. After lengthy but straightforward calculations, we get the solution

$$
\begin{aligned}
A_{s}(x, t)= & {\left[1+(1 / 2) F_{3}\left|S_{s t}\right|^{2}\right] S_{s t} \exp i\left(\omega_{c} t+k_{c} x\right) } \\
& +\left[1-(1 / 2) F_{3}^{*}\left|S_{s t}\right|^{2}\right] S_{s t}^{*} \exp -i\left(\omega_{c} t+k_{c} x\right),
\end{aligned}
$$

where $F_{3}$ is defined as $\operatorname{Re}\left(F_{3}\right)=2 C D / N_{\mathrm{DE}}$ and $\operatorname{Im}\left(F_{3}\right)$ $=2 \Delta_{p} /\left(1+\Delta_{p}^{2}\right)+C\left(1+C^{2}-D^{2}\right) / N_{\mathrm{DE}} \quad$ with $\quad N_{\mathrm{DE}}=[1+(C$ $\left.+D)^{2}\right]\left[1+(C-D)^{2}\right]$. Note that the spatial modulations of these traveling waves are not relevant here and have been neglected (i.e., $k=0$ ) in writing the above solution that is still composed of two asymmetric nonlinear traveling waves. The nonlinear symmetry breaking depends on the set of parameters in which the DOPO operates via the ratio between the intensities [i.e., $R^{2}=\left|A_{s}^{2}\left(k_{c}\right)\right| /\left|A_{s}^{2}\left(-k_{c}\right)\right|=I\left(k^{c}\right) / I\left(-k^{c}\right)$ ] of the two transverse modes of the signal [Eq. (4)]. This ratio has the explicit form

$$
R^{2}=1+\frac{\operatorname{Re}\left(F_{3}\right)}{\operatorname{Re}(\beta)} \frac{2}{u_{1}^{2}+u_{2}^{2}}(\mu-1)
$$

with $u_{1}=1-\left[\operatorname{Re}\left(F_{3}\right) / 2 \operatorname{Re}(\beta)\right](\mu-1)$ and $u_{2}=\left[\operatorname{Im}\left(F_{3}\right) /\right.$ $2 \operatorname{Re}(\beta)](\mu-1)$.

This is the main analytical result. It allows a quantitative characterization of the nonlinear convection effects. Equation (5) emphasizes the coupling between convection and the distance from threshold. In the absence of convection $R^{2}=1$, the two transverse modes have the same intensity and the amplitude equation exhibits stationary rolls. The presence of convection greatly complicates the expression of $R^{2}$. However, near threshold $(\mu \gtrsim 1)$, the ratio of intensities $R^{2}$, up to the leading order in $\mu-1$, is

$$
R^{2} \simeq 1+4 \frac{C\left(1+\Delta_{p}^{2}\right)}{2 N_{\mathrm{DE}}+\left(1+\Delta_{p}^{2}\right)\left(1+C^{2}+D^{2}\right)} D(\mu-1) .
$$

Note that $R^{2}-1$ is an odd function of $\alpha_{s}$, reflecting the importance of the sign of the velocity convection. Therefore, the choice of the convection direction $\left( \pm \alpha_{s}\right)$ can be useful to select one of the two modes by enhancing its parametric gain. Figure 1 shows a typical variation of $R^{2}$ upon the physical pump amplitude $E_{0}=\mu \sqrt{1+\Delta_{p}^{2}}$. We find a very good agreement between the analytical ratio $R^{2}$ and the numerical simulations of the Eqs. (1). In order to set the validity range 


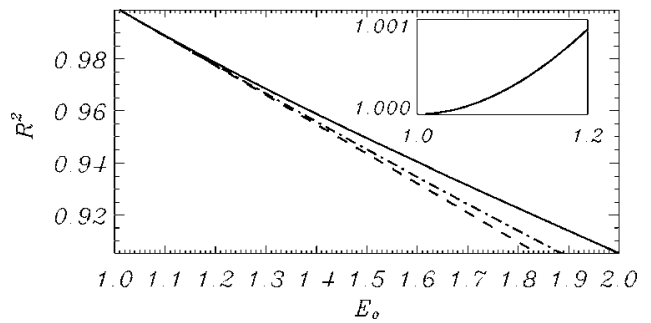

FIG. 1. Dependence of $R^{2}$ on the pump parameter $E_{0}$ above threshold $\left(E_{0}^{c}=1\right)$. Numerical data obtained from integration of Model (1) (continuous line) compared with predictions of Eq. (5) (dashed-dotted line) and Eq. (6) (dashed line). The inset shows the ratio between the continuous and the dashed-dotted lines. The parameters are: $\gamma_{p}=\gamma_{s}=1, \Delta_{p}=0, \Delta_{s}=-1, a_{p}=0.25, a_{s}=0.5$, and $\alpha_{s}$ $=0.25$.

of our predictions we have plotted the results obtained by increasing the pump until twice the threshold. Even for pump values $20 \%$ above threshold the agreement is within $1 \%$ o (see inset of Fig. 1).

The ratio between the intensities of the two critical modes also provides the quantitative characterization of the effects of the convection strength in the nonlinear symmetry breaking. The numerical and analytical estimations of $R^{2}$ versus convection parameter $\alpha_{s}$, displayed in Fig. 2, are again in very good agreement. Finally, note in Fig. 2 the existence of extrema leading to the most asymmetric configuration.

Let us now comment on the physics underlying the nonlinear symmetry breaking induced by convection. The most relevant physical parameter in the nonlinear interaction, above threshold, stems from the difference in frequencies of oscillations of each mode $\left( \pm k_{c}\right)$. This difference results from the presence of convection and disappears with it. Although both traveling waves are propagating in the direction of the convection, their phase rotation is no more opposite. Hence, the two traveling modes interact with a time delay with the pump. This gives rise to different gain from the pump leading to the nonlinear symmetry breaking observed in the signal. The energy transfer depends on two time scales and thus involves the pump decay rate $\left(\gamma_{p}\right)$ as can be seen from the expression of $D$. We emphasize that in contrast with all previous studies dealing with the weakly nonlinear dynamics of optical parametric oscillators near threshold, $\gamma_{p}$ appears, in the cubic Ginzburg-Landau model because of the induced

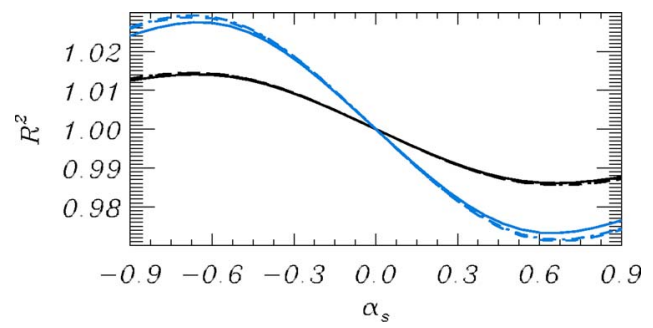

FIG. 2. (Color online) Dependence of $R^{2}$ on convection $\left(\alpha_{s}\right)$. Numerical data (continuous line) are compared with Eq. (5) (dashed-dotted line) and Eq. (6) (dashed line). $E_{0}=1.05 E_{0}^{c}$ (dark lines), $E_{0}=1.1 E_{0}^{c}$ (light lines), $\gamma_{p}=\gamma_{s}=1, \Delta_{p}=-0.2, \Delta_{s}=-0.5, a_{p}$ $=0.5, a_{s}=1$.



FIG. 3. Analytical velocity given by Eq. (7) (continuous line) compared with numerical results (symbols) from the original model (1). In the inset plot we compare the velocities far from threshold. Same parameters of Fig. 1.

pump excitation phenomenon. This fixes the parameter range of the pump decay rates leading to a nonlinear symmetry breaking in the generated traveling waves. The stronger the pump decay rate, the weaker the asymmetry is. In the limit of adiabatic elimination of the pump, no asymmetry exists in the signal, consistent with the possibility to remove the walkoff by a change of reference frame. We have performed numerical simulations (not shown) with the same parameters as in Fig. 1 except that $\gamma_{p}$ is decreased ten times. In this case we have observed a vanishing asymmetry $\left(R^{2} \simeq 1\right)$ with respect to the result of Fig. 1.

The second important feature that results from the convection-induced nonlinear symmetry breaking concerns the propagation velocity of the generated traveling waves. Indeed, the convection effect on the signal is not only a translation of its transverse profile at the convection velocity. An increase in the pump enhances the action of convection in the nonlinear coupling between the fields leading to the velocity variation with the pump intensity. So that, if we set $\gamma_{s} \Omega=\omega_{\text {cor }}$, the corrected frequency of the traveling waves is $\omega_{R}=\omega_{c}+\omega_{c o r}$. Therefore, their actual velocity is given by

$$
v=\frac{\omega_{c}+\gamma_{s} \Omega}{k_{c}}=-\alpha_{s} \gamma_{s}-\gamma_{s} \frac{\operatorname{Im}(\beta)}{k_{c} \operatorname{Re}(\beta)}(\mu-1) .
$$

The above velocity expression shows that, in addition to the usual convection velocity (the first term of the right-hand side) there is an excess velocity depending on the convection but, and most interestingly, it depends linearly on the incident pump above threshold $(\mu-1)$. Figure 3 shows the predicted deviation of the actual velocity from the velocity convection by integrating the full nonlinear equations governing the DOPO dynamics [Eqs. (1)] when increasing the pump $E_{0}$ until twice the threshold. As can be seen from this figure, there is a very good quantitative agreement for a pump until $10 \%$ above threshold. Only at threshold do the nonlinear wave velocities coincide with the convection velocity.

To summarize, we have shown, in case of a degenerate optical parametric oscillator, that convection (walk-off) induced a nonlinear symmetry breaking in the traveling waves. We have also demonstrated that near threshold this mechanism is still described by a Ginzburg-Landau model with an original dependence of the nonlinear self-coupling term upon convection. As a result, nonlinear traveling waves are no 
more symmetrical and the explicit analytical expressions of their intensity variations with both convection and the distance from threshold are derived. Moreover, convection leads to nonlinear phase modulations that give rise to an interesting variation of the traveling wave's velocity with the distance from threshold. Besides the context of optics, our results are relevant to many spatially nonlinear extended systems with convection. For instance, in the context of hydrodynamics, the competition between right- and leftpropagating nonlinear waves in the convective flow, generated by a horizontal thermal gradient, leads to an experimental observation of the nonlinear symmetry break- ing. The broken symmetry has been evidenced via the estimation of the variation of the amplitude ratio of the right and left waves with the distance from threshold [17].

M.T. and C.D. acknowledge financial support from the Laboratoire de Physique des Lasers, Atomes et Molécules, and CNRS. R.Z. acknowledges financial support from the UK Engineering and Physical Sciences Research Council (GR/S03898/01). MSM acknowledges financial support from the Spanish MED through projects FIS2004-00953 and BFM2001-4180-E.
[1] M. Cross and P. C. Hohenberg, Rev. Mod. Phys. 65, 851 (1993); J. D. Murray, Mathematical Biology (Springer, Berlin, 1993).

[2] E. Infeld and G. Rowlands, Nonlinear Waves, Solitons and Chaos (Cambridge University Press, Cambridge, UK, 1990).

[3] L. A. Lugiato, M. Brambilla, and A. Gatti, in Advances in Atomic, Molecular and Optical Physics, edited by B. Bederson and H. Walther, (Academic, New York, 1998), Vol. 40; W. Lange and T. Ackermann, J. Opt. B: Quantum Semiclassical Opt. 2, 347 (2000); K. Staliunas and V. J. Sanchez-Morcillo, Transverse Patterns in Nonlinear Optical Resonators (Springer-Verlag, Berlin, 2003); P. Mandel and M. Tlidi, J. Opt. B: Quantum Semiclassical Opt. 6, R60 (2004).

[4] N. N. Rosanov, in Spatial Hysteresis and Optical Patterns (Springer, Berlin, 2002).

[5] P. Huerre and P. A. Monkewitz, Annu. Rev. Fluid Mech. 22, 473 (1990); A. Couairon and J. M. Chomaz, Phys. Rev. Lett. 79, 2666 (2000); H. R. Brand, R. J. Deissler, and G. Ahlers, Phys. Rev. A 43, 4262 (1991); P. Büchel and M. Lucke, Phys. Rev. E 61, 3793 (2000); X. Nicolas, A. Mojtabi, and J. K. Platten, Phys. Fluids 9, 337 (1997); L. Pastur, M. T. Westra, and W. van de Water, Physica D 174, 71 (2003).

[6] R. J. Briggs, Electron-Stream Interaction with Plasmas (MIT Press, Cambridge, MA, 1964).

[7] N. Mitarai and H. Nakanishi, Phys. Rev. Lett. 85, 1766 (2000).

[8] N. Israeli, D. Kandel, M. F. Schatz, and A. Zangwill, Surf. Sci.
494, L735 (2001).

[9] M. Santagiustina, P. Colet, M. San Miguel, and D. Walgraef, Phys. Rev. Lett. 79, 3633 (1997); G. Izùs, M. Santagiustina, M. San Miguel, and P. Colet, J. Opt. Soc. Am. B 16, 1592 (1999); H. Ward, M. Taki, and P. Glorieux, Opt. Lett. 27, 348 (2002); E. Louvergneaux, C. Szwaj, G. Agez, P. Glorieux, and M. Taki, Phys. Rev. Lett. 92, 043901 (2004); S. Coen, M. Tlidi, P. Emplit, and M. Haelterman, Phys. Rev. Lett. 83, 2328 (1999).

[10] M. Santagiustina, P. Colet, M. San Miguel, and D. Walgraef, Phys. Rev. E 58, 3843 (1998); H. Ward, M. N. Ouarzazi, M. Taki, and P. Glorieux, Eur. Phys. J. D 3, 275 (1998).

[11] R. Zambrini, S. M. Barnett, P. Colet, and M. San Miguel, Eur. Phys. J. D 22, 461 (2003).

[12] R. Zambrini, S. M. Barnett, P. Colet, and M. San Miguel, Phys. Rev. A 65, 023813 (2002).

[13] R. Zambrini and M. San Miguel, Phys. Rev. A 66, 023807 (2002).

[14] H. Ward, M. N. Ouarzazi, M. Taki, and P. Glorieux, Phys. Rev. E 63, 016604 (2001); S. Longhi, Phys. Rev. A 53, 4488 (1996).

[15] G.-L. Oppo, M. Brambilla, and L. A. Lugiato, Phys. Rev. A 49, 2028 (1994).

[16] S. Fauve and O. Thual, Phys. Rev. Lett. 64, 282 (1990).

[17] N. Garnier and A. Chiffaudel, Phys. Rev. Lett. 86, 75 (2001). 